\title{
Heartbeat: sex disparities in stroke, heart failure and all-cause mortality in adults with coronary heart disease
}

Research on sex disparities in patients with coronary heart disease (CHD) largely has focused on events directly related to the coronary arteries themselves-recurrent myocardial infarction, revascularisation and cardiovascular death-rather than the wider consequences of atherosclerotic vascular disease. In contrast, Akyea and colleagues ${ }^{1}$ evaluated sex disparities across a broader range of major adverse cardiovascular events (MACE) including not only recurrent $\mathrm{CHD}$, but also stroke, peripheral vascular disease, heart failure and all-cause mortality in a population-based cohort of 143702 adults (median age 73 years in women and 66 years in men) with no prior cardiovascular events. MACE occurred in 63.8\% and recurrent $\mathrm{CHD}$ in $46.3 \%$ with lower risks of composite MACE (HR 0.68 (95\% CI 0.67 to 0.69 ) or recurrent $\mathrm{CHD}$ (HR, 0.60 (0.59 to 0.61$)$ in women compared with men. However, women had a higher risk of stroke (4\%, HR, 1.26 (1.19 to 1.33), heart failure (5.5\%, HR, 1.09 (1.04 to 1.15$)$ and all-cause mortality $(20.5 \%$, HR, 1.05 (1.02 to 1.07), with an older age distributin of events compared with men (figure 1).

In the accompanying editorial, Asleh ${ }^{2}$ argues that is 'time to move from observation to action' in addressing sex disparities in outcomes after a CHD event. Asleh suggests the following strategies to address these disparities:

- Increase representation of women in preclinical and clinical studies.

- Elucidate further mechanisms response for sex differences in coronary physiology.

- Integrate psychosocial, cultural, race/ ethnicity and physiological factors in study designs.

- Improve primary and secondary prevention strategies.

- Educate for seeking early management and improving adherence to medical therapy.

Division of Cardiology, University of Washington, Seattle, Washington, USA

Correspondence to Professor Catherine M Otto, Division of Cardiology, University of Washington, Seattle, WA 98195, USA; cmotto@uw.edu
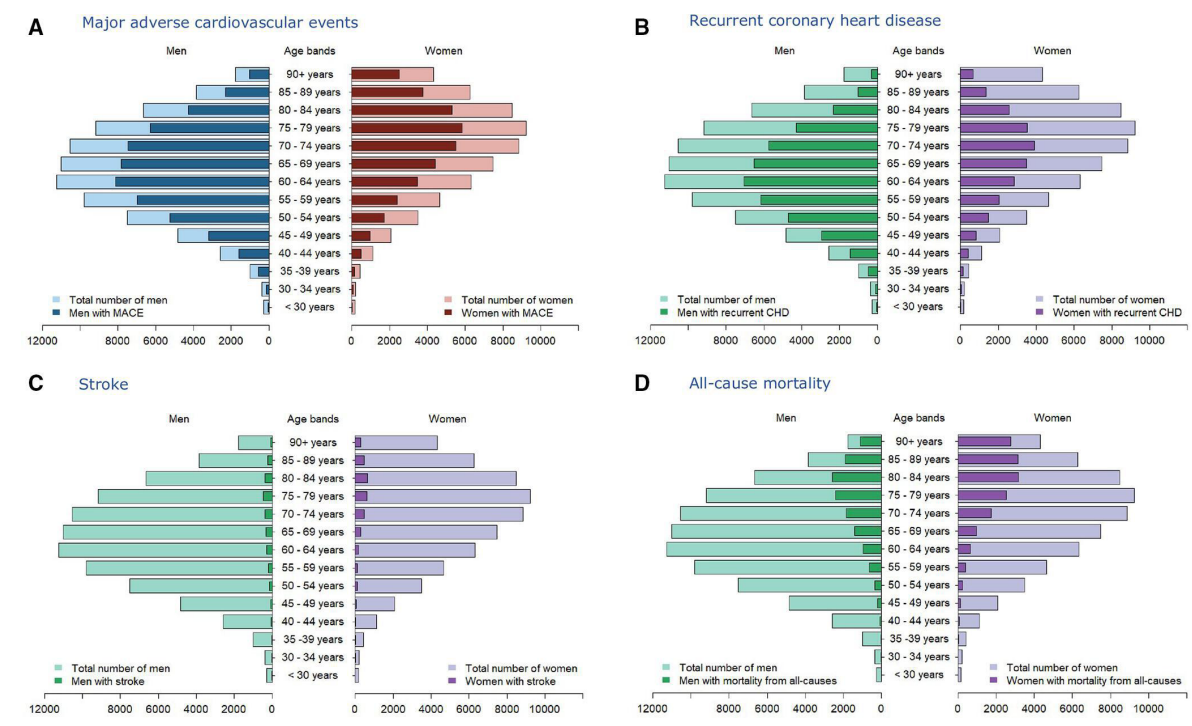

D All-cause mortality

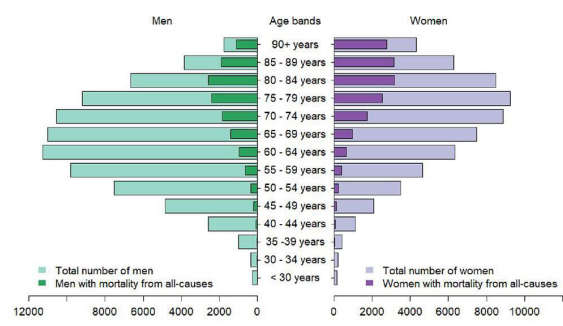

Figure 1 Distribution of first subsequent major adverse outcomes by sex and 5 year age group for patients with incident CHD. CHD, coronary heart disease; MACE, major adverse cardiovascular events.

- Develop sex-specific algorithms for risk stratification and management of CHD.'
No doubt these strategies would improve outcomes for both women and men with CHD in the long term.

As we perform transcatheter aortic valve implantation (TAVI) for

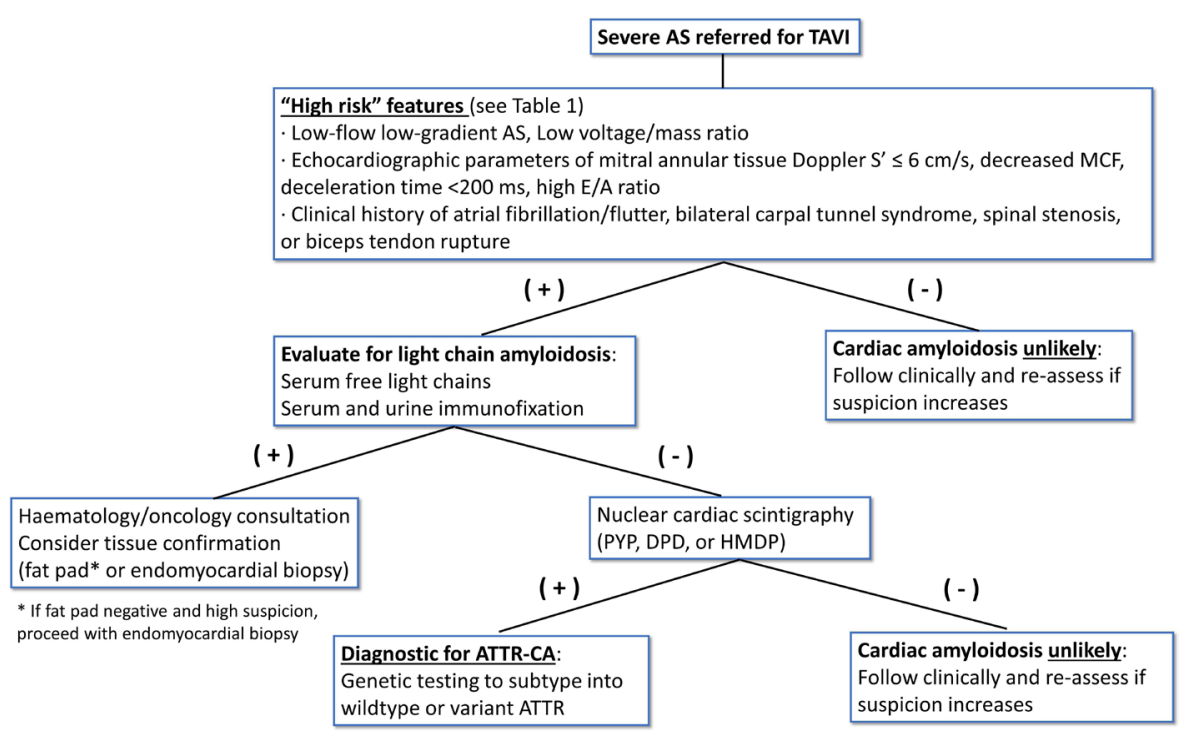

Figure 2 Proposed algorithm for screening older patients with severe AS referred for TAVI for underlying cardiac amyloidosis. AS, aortic stenosis; ATTR, transthyretin cardiac amyloidosis; CA, cardiac amyloidosis; DPD, 3,3-diphosphono-1,2-propanodicarboxylic acid; HMDP, ${ }^{99 \mathrm{~m}} \mathrm{Tc}$ hydroxymethylene diphosphonate; MCF, myocardial contraction fraction; PYP, ${ }^{99 \mathrm{~m}} \mathrm{Tc}$ pyrophosphate; TAVI, transcatheter aortic valve implantation. 


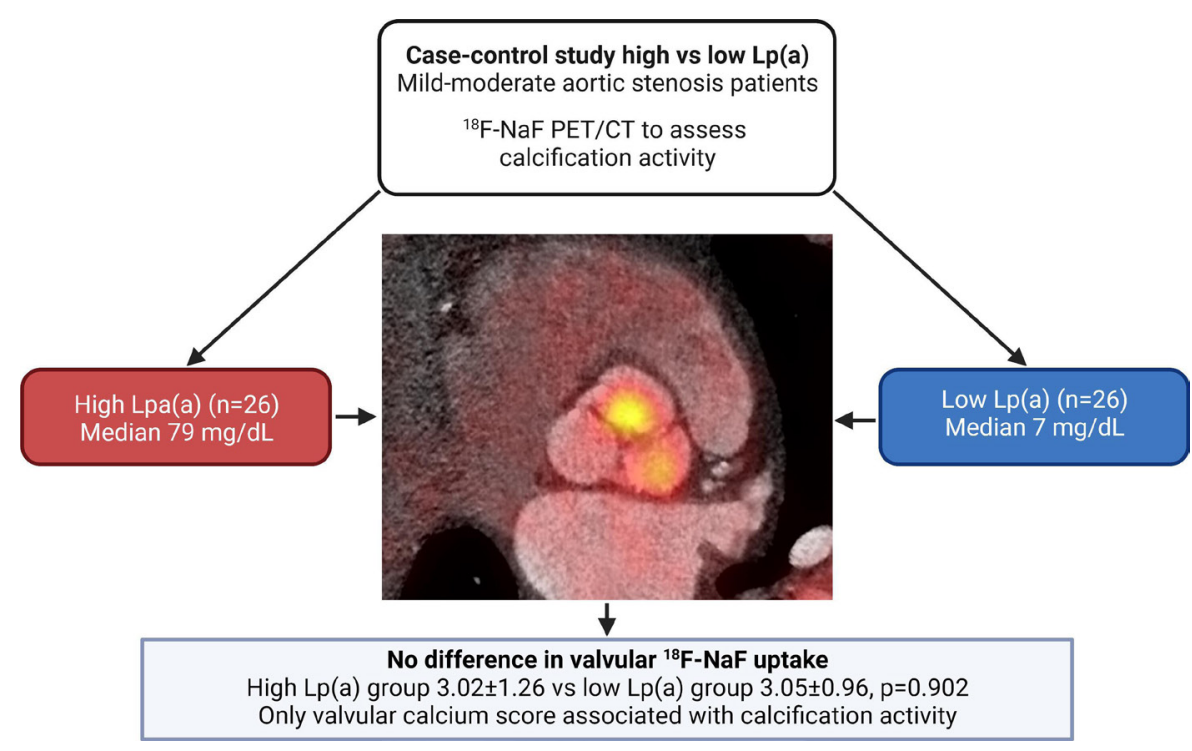

Figure $3 \mathrm{Lp}(\mathrm{a})$ has no major impact on calcification activity in patients with mild to moderate aortic valve stenosis. in this case-control study consisting of matched patients with aortic stenosis with high versus low Lp(a), we observed comparable calcification activity in both groups. Aortic valve calcium score was the only variable associated with ${ }^{18} \mathrm{~F}-\mathrm{NaF}$ uptake in linear regression analysis ( $\beta=0.60$ per 1000 Agatston unit increase, $95 \% \mathrm{Cl} 0.39$ to 0.81 ). Lp(a), lipoprotein(a); $\mathrm{NaF}$ $\mathrm{PET} / \mathrm{CT}$, sodium fluoride positron emission tomography.

symptomatic severe aortic stenosis (AS) to older and sicker patients, it has become evident that many patients with AS have concurrent wild-type transthyretin cardiac amyloidosis (ATTR) which may account for persistent symptoms after TAVI. Patel and colleagues ${ }^{3}$ compared cardiac remodelling, ventricular function and serum markers in 359 patients with AS alone, 107 with ATTR alone, 35 with both AS and ATTR and 81 matched controls using multimodality imaging, including nuclear scintigraphy. Overall, they found that patients with combined AS and ATTR were similar to those with ATTR alone in terms of carpal tunnel symptoms and diastolic dysfunction, although LV mass was lower in those with AS. Both patients with AS and ATTR (or the combination) had

abnormal left ventricular global longitudinal strain and right ventricular annular motion, consistent with early biventricular systolic dysfunction.

Cheng and Griffin ${ }^{4}$ point out that the high prevalence of concurrent AS and ATTR is not surprising given that 'Approximately 25\% of people over 85 years of age have ATTR deposition in the myocardium at autopsy, while severe AS affects $>3 \%$ of individuals over 75 years.' In addition, 'the two disease entities share common features, including older age, increased left ventricular (LV) wall thickness, diastolic dysfunction and elevated natriuretic peptides.' Because treatment of ATTR may improve outcomes after TAVI in patients with combined AS-ATTR, Cheng and Griffin ${ }^{4}$ recommend screening in high-risk patients (figure 2).
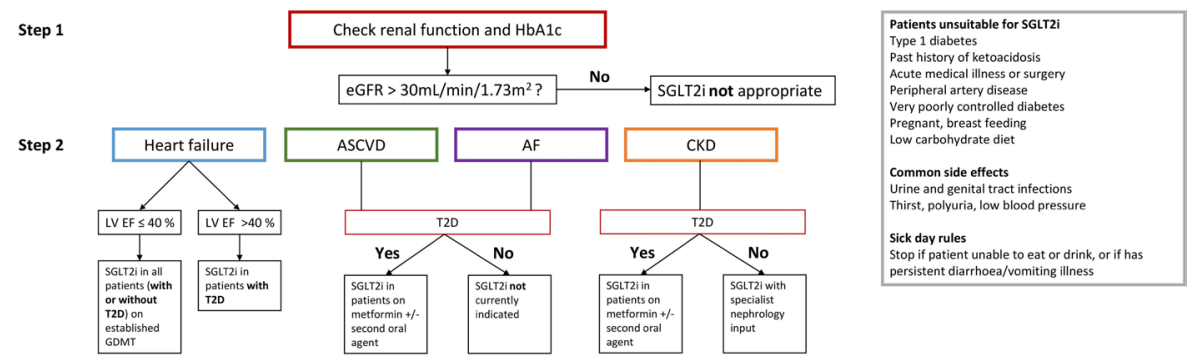

Step 3

Re-check renal function $4-6$ weeks after initiation and continue if $<10 \%$ decline

Figure 4 Guide for patient selection when initiating SGLT2i. AF, atrial fibrillation; ASCVD, atherosclerotic cardiovascular disease; CKD, chronic kidney disease; EF, ejection fraction; eGFR, estimated glomerular filtration rate; GDMT, guide-directed medical therapy; $\mathrm{HbA} 1 \mathrm{c}$, haemoglobin A1C; LV, left ventricular; T2D, type 2 diabetes; SGLT2i, sodium glucose cotransporter two inhibitor.
Another interesting paper in this issue of Heart addressed the hypothesis that high lipoprotein(a) levels (Lp(a)) would be associated with increased calcification activity in the aortic valve leaflets. Kaiser and colleagues ${ }^{5}$ performed ${ }^{18} \mathrm{~F}$-sodium fluoride $\left({ }^{18} \mathrm{~F}-\mathrm{NaF}\right)$ positron emission tomography/CT in 26 matched pairs of patients with mild to moderate AS (peak aortic jet velocity between 2 and $4 \mathrm{~m} / \mathrm{s}$ ) and high versus low $\mathrm{Lp}(\mathrm{a})$ (>50 mg/dL vs $<50 \mathrm{mg} / \mathrm{dL}$ ) (figure 3 ). However, the data do not support the author's hypothesis and instead suggest that calcification activity is primarily related to the degree of calcification at baseline, not to serum $\mathrm{Lp}(\mathrm{a})$ levels.

These results seem surprising given the numerous studies showing an association between serum $\operatorname{Lp}(\mathrm{a})$ and the incidence of aortic valve calcification (AVC), as well as genome wide studies showing that polymorphisms in $\mathrm{Lp}(\mathrm{a})$ are associated with AVC. Chan ${ }^{6}$ proposes that 'these contradictory findings can be reconciled in light of the pathophysiology of AS which consists of three phases (the initiation, propagation and end-stage calcification phases) during which different specific metabolic pathways may be dominant.' He goes on to note that 'the presence of AVC represents a late stage of the disease which is likely the reason why trials on lipid lowering to prevent AS progression so far have been unsuccessful. Prevention approaches targeting lipids including $\mathrm{Lp}$ (a) may be more effective in individual at risk for AS such as individuals with bicuspid aortic valve before the development of significant AVC. When AVC is present, strategies targeting specific mediators of the calcification process appear more appropriate and we await the results of the ongoing trials based on this approach.'

The Education in Heart article ${ }^{7}$ in this issue discusses the increased risk of atherosclerotic vascular disease in patients with systemic inflammatory diseases, focusing on the three most common - rheumatoid arthritis, spondylarthritis and inflammatory bowel disease-which affect 5\%-7\% of the population worldwide. Risk reduction in these patients includes both assessment and treatment of conventional risk factors in conjunction with ensuring optimal anti-inflammatory therapy, with close communication between the cardiology and rheumatology teams in coordination of care.

Another useful article in this issue summarises the use of sodium glucose cotransporter 2 (SGLT2) inhibitor medications in patients with cardiovascular disease. ${ }^{8}$ The SGLT2 inhibitors reduce 
mortality and hospitalisations in patients with heart failure and in those with Type 2 diabetes complicated by atherosclerotic vascular disease, atrial fibrillation or chronic kidney disease. This article provides practical guidance for cardiologists in identifying patients who might benefit for this newer therapy and avoiding situations in which SGLT2 inhibitor therapy is not appropriate (figure 4).

Funding The authors have not declared a specific grant for this research from any funding agency in the public, commercial or not-for-profit sectors.

Competing interests None declared.

Patient and public involvement Patients and/or the public were not involved in the design, or conduct, or reporting, or dissemination plans of this research.

Patient consent for publication Not applicable.
Provenance and peer review Commissioned; internally peer reviewed.

(c) Author(s) (or their employer(s)) 2022. No commercial re-use. See rights and permissions. Published by BMJ.

\section{A Check for updates}

To cite Otto CM. Heart 2022;108:1-3.

Heart 2022;108:1-3.

doi:10.1136/heartjnl-2021-320617

\section{ORCID iD}

Catherine M Otto http://orcid.org/0000-0002-05279392

\section{REFERENCES}

1 Akyea RK, Kontopantelis E, Kai J. Sex disparity in subsequent outcomes in survivors of coronary heart disease. Heart 2022;108:37-45.
2 Asleh R. Persistent sex differences in outcomes after coronary heart disease: time to move from observation to action. Heart 2022;108:4-6.

3 Patel KP, Scully PR, Nitsche C. Impact of afterload and infiltration on coexisting aortic stenosis and transthyretin amyloidosis. Heart 2022:108:67-72.

4 Cheng R, Griffin J. Implications of screening for coexisting transthyretin amyloidosis and aortic stenosis. Heart 2022;108:11-13.

5 Kaiser Y, Nurmohamed NS, Kroon J. Lipoprotein(a) has no major impact on calcification activity in patients with mild to moderate aortic valve stenosis. Heart 2022:108:61-6.

6 Chan K-L. Lipoprotein(a) and aortic stenosis. Heart 2022;108:9-10.

7 Agca R, Smulders Y, Nurmohamed M. Cardiovascular disease risk in immune-mediated inflammatory diseases: recommendations for clinical practice. Heart 2022:108:73-9.

8 Gulsin GS, Graham-Brown MPM, Squire IB. Benefits of sodium glucose cotransporter 2 inhibitors across the spectrum of cardiovascular diseases. Heart 2022;108:16-21. 